\title{
A genetic bistable switch utilizing nonlinear protein degradation
}

\author{
Daniel Huang ${ }^{1}$, William J Holtz ${ }^{2}$ and Michel M Maharbiz ${ }^{1 *}$
}

\begin{abstract}
Background: Bistability is a fundamental property in engineered and natural systems, conferring the ability to switch and retain states. Synthetic bistable switches in prokaryotes have mainly utilized transcriptional components in their construction. Using both transcriptional and enzymatic components, creating a hybrid system, allows for wider bistable parameter ranges in a circuit.

Results: In this paper, we demonstrate a tunable family of hybrid bistable switches in E. coli using both transcriptional components and an enzymatic component. The design contains two linked positive feedback loops. The first loop utilizes the lambda repressor, $\mathrm{Cl}$, and the second positive feedback loop incorporates the Lon protease found in Mesoplasma florum ( $m f$-Lon). We experimentally tested for bistable behavior in exponential growth phase, and found that our hybrid bistable switch was able to retain its state in the absence of an input signal throughout 40 cycles of cell division. We also tested the transient behavior of our switch and found that switching speeds can be tuned by changing the expression rate of $\mathrm{mf}$-Lon.

Conclusions: To our knowledge, this work demonstrates the first use of dynamic expression of an orthogonal and heterologous protease to tune a nonlinear protein degradation circuit. The hybrid switch is potentially a more robust and tunable topology for use in prokaryotic systems.
\end{abstract}

\section{Introduction}

As is well known, bistability is a fundamental property of a vast array of engineered and natural systems. Bistability confers the ability to switch, to retain state, and to store information, all three comprising a closely related set of operations common to computational systems. Not surprisingly, bistable networks and components are found to be central to many cellular processes, ranging from cell cycle and differentiation, to cell fate determinations and environmental sensing [1-6]. Over the last decade, a great deal of interest has arisen towards the creation of synthetic bistable genetic systems for use as memories, decision making circuits or sustained response devices [7-9]. Many studies have focused on understanding and improving these systems, and creating proper design rules for building synthetic bistable switches and modifying existing systems [10-13].

The most basic bistable switch is a single positive feedback loop. Bistable switches consisting of a single positive

\footnotetext{
* Correspondence: maharbiz@eecs.berkeley.edu

${ }^{1}$ Department of Electrical Engineering and Computer Science, University of California, 656 Sutardja Dai Hall,Berkeley, Berkeley, CA94720, USA

Full list of author information is available at the end of the article
}

feedback loop are rarely observed in nature. The first synthetic bistable genetic switch was a single positive feedback loop constructed with a pair of co-antagonistic repressors [14]. Even in the absence of cooperativity, a single positive feedback loop containing a transcriptional activator and its cognate promoter can exhibit bistable behavior due to transcriptional nonlinearity from cell growth [15]; employing variations resulting from cell growth, however, has proven difficult to exploit. Both natural and synthetic systems often contain multiple feedback loops for added control and robustness $[10,11,16]$.

Protein degradation rates have also been mentioned to be critical in the operation of bistable and bimodal networks [17]; bimodal gene expression can result simply from changing protein degradation rates $[18,19]$. The Vibrio fisheri quorum sensing system is an example from nature that uses nonlinear degradation to create a bistable system. Here nonlinear degradation is achieved through cooperative stability, where proteins are protected against degradation by protein multimerization. The positive transcription factor LuxR turns on the $l u x$ promoter, producing more LuxR. LuxR as a monomer is unstable and has a fast degradation rate. The LuxR monomer does not

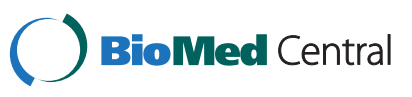


accumulate to high concentrations and also cannot activate the lux promoter. In the presence of the autoinducer AHL, LuxR dimerizes into a stable and slowdegrading complex. The LuxR-AHL complex is highly stable, is able to accumulate to a high concentration and is also able to activate the lux promoter. Varying the levels of AHL can switch the lux promoter between "on" and "off" states. Unfortunately, creating a synthetic bistable system utilizing cooperative stability is difficult, requiring protein engineering to create synthetic proteins with cooperative stability.

Nonlinear degradation conditions can also be achieved through dynamic protein degradation. Dynamic protein degradation has been observed in Xenopus, and is responsible for cell cycle progression. The concentration of Cdc2-cyclin B increases during interphase, but abruptly drops during mitosis. The accumulation of $\mathrm{Cdc} 2$-cyclin $\mathrm{B}$ actives the anaphase promoting complex (APC), and the APC inactivates Cdc2-cyclin B through proteolysis. A mitotic oscillator is observed in Xenopus during the early embryonic cell cycle [20], by taking advantage of dynamic degradation of Cdc2-cyclin B.

All reported synthetic bistable switches implemented in prokaryotes exclusively use transcriptional components (i.e. transcriptional activators, repressors and their cognate promoters). However, bistable systems can, in principle, be constructed from diverse components and their interactions: transcriptional elements, enzymes, transport proteins, metabolic pathways, ligand binding, etc. A recent report compared bistable switches constructed with enzymatic feedback loops, feedback loops constructed from only transcriptional components and hybrid transcriptional/enzymatic switches [12]. It was found that enzyme-only circuits and hybrid transcriptional/enzymatic circuits showed bistable behavior for a wider parameter range than transcription-only circuits. This implies that hybrid bistable systems would likely be easier to tune and be more robust than systems built only from existing transcriptional components. In natural prokaryotic systems, the lac operon is arguably the most well-studied hybrid transcriptional/enzymatic bistable switch [21-23]. This system incorporates a lactose permease, enzymatic reactions, and transcriptional regulation. Once again, another well-studied hybrid bistable system is the lux operon in Vibrio fischeri [24], which uses an enzymatic reaction to convert a small molecule into an autoinducer to trigger transcriptional activation.

In this paper, we demonstrate a tunable family of hybrid bistable switches in E. coli using both transcriptional components and an enzymatic component (protease). We utilize standard transcriptional components to create a positive feedback loop. The behavior of the positive feedback loop, which normally does not show bistable behavior, is changed to a bistable network by adding a second feedback loop incorporating the Lon protease found in Mesoplasma florum ( $m f$-Lon). To our knowledge, this work demonstrates the first use of $m f$ Lon to tune a nonlinear protein degradation circuit.

\section{Theory and design \\ Dynamic protein degradation and bistability}

In this paper, we demonstrate the use of dynamic protein degradation to create a bistable switch. The switch (Figure1a) employs the Lon protease found in Mesoplasma florum (mf-Lon). This protease specifically degrades appropriately tagged proteins [25]. Mf-Lon works in much the same way as the more familiar E. coli ssrA degradation system, which utilizes the proteins ClpX and ClpP [26]. When translation stalls in E. coli, an ssrA tag is added to the C-terminus of the stalled polypeptide, which targets the polypeptide for degradation. Once the polypeptide is degraded, the ribosome is free to continue translation. The native E. coli degradation tag (ec-ssrA) is 13 amino acids long, while the M. florum degradation tag ( $m f$-ssrA) is 32 amino acids. These two tags appear to be fully orthogonal and only the cognate proteases can recognize the proper tag [25]. $M f$-Lon has been shown to function properly in E. coli, by degrading LacZ tagged with $m f$-ssrA [25]. We thus hypothesized that $m f$-Lon could be used in E. coli for dynamically targeting protein degradation by tagging specific proteins with $m f$-ssrA and dynamically expressing $m f$-Lon.

Other methods for engineering dynamic protein degradation exist but have disadvantages. In E. coli, knocking out the proteins $\mathrm{ClpX}$ and $\mathrm{ClpP}$ for dynamic expression compromises the fitness of knockout strains [27]. B. subtilis has a similar ssrA system as E. coli [28] and utilizes the same ssrA tags. Dynamic degradation of tagged proteins is possible in B. subtilis by using a mutated ssrA tag and dynamic expression of the E. coli gene, $s s b P$ [29]. Proteins tagged with the mutated ssrA tag will only be degraded when $s s b P$ is expressed. This method is plausible for organisms utilizing a native ssrA system similar to $E$. coli but missing the $s s b P$ gene. Unfortunately, these conditions are difficult to meet, and may only be possible with $B$. subtilis. The use of a completely orthogonal tagging and degradation system, such as utilizing $m f$-Lon in E. coli, is thus superior to previously mentioned methods for engineering dynamic degradation of protein.

\section{Design of circuit}

The switch is a hybrid of transcriptional components and enzymatic degradation (Figure 1a). It is comprised of two positive feedback loops: a positive auto-regulating loop and a loop with two antagonistic interactions (Figure 1b). Our positive auto-regulating loop is identical 


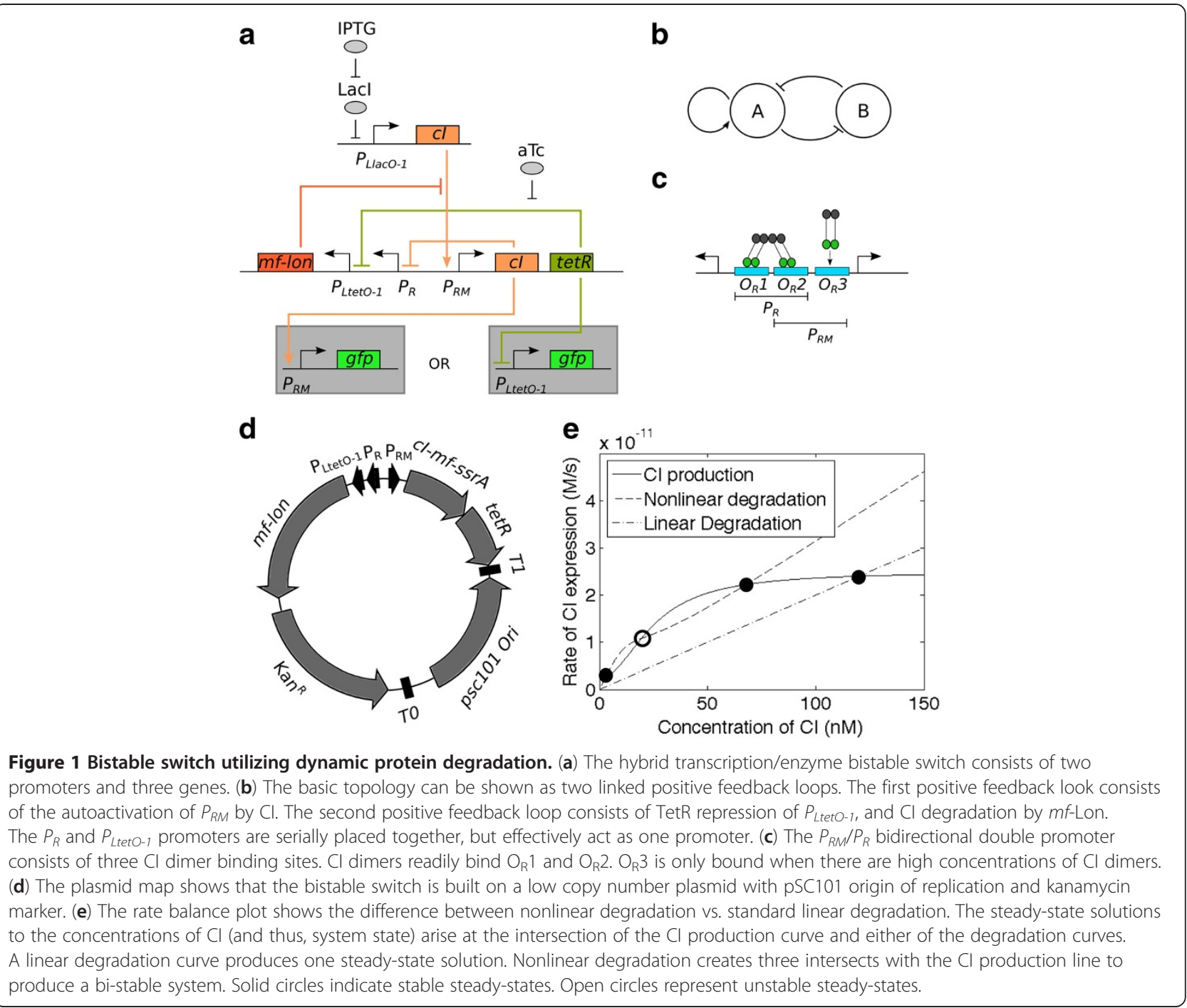

to one found in $\lambda$ phage. The promoter $\mathrm{P}_{R M}$ expresses the $\lambda$ repressor $\mathrm{CI}$. CI is a transcriptional activator of $\mathrm{P}_{R M}$, thus producing positive feedback. The second positive feedback loop uses the tetracycline repressor (TetR) and $m f$-Lon. TetR is expressed bicistronically with $\mathrm{CI}$ in the $\mathrm{P}_{R M^{-}} \mathrm{CI}$ loop. $\mathrm{CI}$ in the autoregulated loop is tagged with $m f$-ssrA (CI- $m f$-ssrA), allowing for fast degradation in the presence of $m f$-Lon. $\mathrm{P}_{\text {LtetO-1 }}$ promoter [30] controls $m f$-Lon production. TetR is a repressor of $\mathrm{P}_{\text {LtetO-1 }}$ and can stop $m f$-Lon expression, thus creating the coantagonistic interaction; $m f$-Lon expression shuts off $\mathrm{CI}$ and TetR expression, while $\mathrm{CI}$ and TetR expression shuts off $m f$-Lon expression.

We defined the "on" versus "off" states of the switch in terms of the $P_{R M^{-}}$CI auto-regulation loop. When CI is produced, the switch is in the "on" state. When CI is degraded, the switch is "off." We used superfolder GFP (sfGFP) [31] tagged with a fast degrading ec-ssrA tag controlled by $\mathrm{P}_{R M}$ (PRM-GFP)(see Table 1 ) to report the state of the switch. We also constructed another reporter plasmid, which expresses sfGFP with $\mathrm{P}_{\text {LtetO-1 }}$ (PLtetO-1GFP). These two reporters were constructed on plasmids with p15A origins of replication and ampicillin markers. Both reporters were separately used to test the switch.

The use of TetR allows for induction using anhydrous tetracycline (aTc). When TetR is bound by aTc, TetR is rendered inactive and can no longer repress $\mathrm{P}_{\text {LtetO-1 }}$ and results in $m f$-Lon expression. This causes CI degradation and forces the switch into the "off" state. CI expression can be forced with IPTG induction using a separate inducible plasmid (PLlacO-1-CImf). Inducing with IPTG forces the switch into the "on" state. Some previous systems incorporating $\mathrm{CI}$ in bistable networks invoked the SOS response to degrade $\mathrm{CI}$ to either flip states or reset the network as $\mathrm{CI}$ is quickly degraded through RecA mediated degradation [14,32]. Unfortunately, methods evoking the SOS response such as heat shock [14] or UV exposure [32] may be detrimental to cultures. Our 
Table 1 List of Plasmids (accession numbers are listed in the supplemental section $\mathbf{S . 5}$ )

\begin{tabular}{|c|c|c|c|}
\hline Name & Description & Marker & Origin \\
\hline PRM-GFP & GFP reporter expressed from $P_{R M}$ & Amp & p15A \\
\hline PLtetO-1-GFP & GFP reporter expressed from $P_{\text {LtetO-1 }}$ & Amp & p15A \\
\hline PLlacO-1-Clwt & IPTG inducible Cl-wt & $\mathrm{Cm}$ & ColE1 \\
\hline PLlacO-1-CILVA & IPTG inducible Cl-ec-ssrA & $\mathrm{Cm}$ & ColE1 \\
\hline PLlacO-1-Clmf & IPTG inducible Cl-mf-ssrA & $\mathrm{Cm}$ & ColE1 \\
\hline RFP1 & $\begin{array}{l}\mathrm{P}_{\text {Lteto-1 }} \mathrm{mf} \text {-lon (trunc) mCherry } \\
\text { with RBS1 }\end{array}$ & Amp & p15A \\
\hline RFP2 & $\begin{array}{l}\mathrm{P}_{\text {Lteto-1 }} \mathrm{mf} \text {-lon (trunc) mCherry } \\
\text { with RBS2 }\end{array}$ & Amp & p15A \\
\hline RFP3 & $\begin{array}{l}\mathrm{P}_{\text {Lteto-1 }} \mathrm{mf} \text {-lon (trunc) mCherry } \\
\text { with RBS3 }\end{array}$ & Amp & p15A \\
\hline RFP4 & $\begin{array}{l}P_{\text {Lteto-1 }} \text { mf-lon (trunc) mCherry } \\
\text { with RBS4 }\end{array}$ & Amp & p15A \\
\hline RFP5 & $\begin{array}{l}P_{\text {Lteto-1 }} \mathrm{mf} \text {-lon (trunc) mCherry } \\
\text { with RBS5 }\end{array}$ & Amp & p15A \\
\hline RFP6 & $\begin{array}{l}P_{\text {Lteto-1 }} \mathrm{mf} \text {-lon (trunc) mCherry } \\
\text { with RBS6 }\end{array}$ & Amp & p15A \\
\hline RFP7 & $\begin{array}{l}P_{\text {Lteto-1 }} \text { mf-lon (trunc) mCherry } \\
\text { with RBS7 }\end{array}$ & Amp & p15A \\
\hline SW1 & Full switch with 5-UTR1 & Kan & pSC101 \\
\hline SW2 & Full switch with 5-UTR2 & Kan & pSC101 \\
\hline SW3 & Full switch with 5-UTR3 & Kan & pSC101 \\
\hline SW4 & Full switch with 5-UTR4 & Kan & pSC101 \\
\hline SW5 & Full switch with 5-UTR5 & Kan & pSC101 \\
\hline SW6 & Full switch with 5-UTR6 & Kan & pSC101 \\
\hline SW7 & Full switch with 5-UTR7 & Kan & $\overline{p S C 101}$ \\
\hline PRM-Clwt-TetR & $\mathrm{Cl}$-wt and TetR expressed from $\mathrm{P}_{R M}$ & Kan & pSC101 \\
\hline PRM-CILVA-TetR & $\begin{array}{l}\text { Cl-ec-ssrA and TetR expressed } \\
\text { from } P_{R M}\end{array}$ & Kan & pSC101 \\
\hline PRM-CImf-TetR & $\begin{array}{l}C l-m f-s s r A \text { and TetR expressed } \\
\text { from } P_{R M}\end{array}$ & Kan & pSC101 \\
\hline PLlacO-1-mfLon & IPTG inducible $m f$-Lon & $\mathrm{Cm}$ & ColE1 \\
\hline
\end{tabular}

system of utilizing $m f$-Lon allows for fast degradation of $\mathrm{CI}$ without evoking the SOS response.

$\mathrm{CI}$ also acts as a negative autoregulator at high concentrations [33] (Figure 1c). $\mathrm{P}_{R M}$ is half of a bidirectional promoter (Figure 1c) in $\lambda$ phage. The complete promoter consists of three binding sites, $\mathrm{O}_{R} 1, \mathrm{O}_{R} 2$ and $\mathrm{O}_{\mathrm{R}} 3$. CI preferentially binds to $\mathrm{O}_{\mathrm{R}} 1$ and $\mathrm{O}_{\mathrm{R}} 2$, repressing the constitutive promoter, $\mathrm{P}_{R}$. In high concentrations, CI binds to $\mathrm{O}_{\mathrm{R}} 3$ and acts as a repressor to $\mathrm{P}_{R M}$. We used a mutated $\mathrm{O}_{\mathrm{R}} 3$ [34], in order to prevent autorepression.

The complete switch was constructed on a plasmid with a pSC101 origin and a kanamycin marker (Figure 1d). Both TetR and $m f$-Lon were tagged with a fast degradation $e c$-ssrA tag to allow for fast turnover rates for the proteins. $\mathrm{P}_{\text {LtetO- } 1}$ has been placed serially next to $\mathrm{P}_{R}$ without separation by a terminator. It is unnecessary to use a terminator between these two promoters, because when the switch is in the "on" state, CI and TetR represses both
$\mathrm{P}_{R}$ and $\mathrm{P}_{\text {LtetO-1 }}$, respectively, and $m f$-Lon production is stopped. When the switch is in the "off" state both $\mathrm{P}_{R}$ and $\mathrm{P}_{\text {LtetO-1 }}$ are not blocked and $m f$-Lon production is resumed. The serial placement of $\mathrm{P}_{\text {LtetO-1 }}$ and $\mathrm{P}_{R}$ should not affect the basic principles governing the design of our switch.

\section{Conceptual comparison of hybrid bistable switch with transcriptional-only switch}

A rate-balance plot [35] highlights the important differences between a fully transcriptional bistable switch (TS) (Supplemental Information S.1.5), and the hybrid transcriptional-enzymatic switch (TES) (Figure 1e). Both a TS and a TES use two linked positive feedback loops. The difference between the two topologies lies in the second positive feedback loop. In the TS, the second positive feedback loop uses two repressors. In the TES, the second positive feedback loop uses a repressor and a protease. The solid line on the rate balance plot (Figure 1e), represents the transcriptional activator production rate and the dashed curves represents the transcriptional activator degradation rate. A monostable system contains only one stable steady-state, represented by one intersection between the protein production and degradation curves. A bistable system requires two stable steady states (closed circles) and one unstable steady state (open circle), for a total of three intersections. In the "classic" TS, the addition of a second positive feedback loop adds nonlinearity to the CI production curve, resulting in a higher apparent Hill coefficient in the CI production curve (Additional file 1: S.1.5). Degradation rates for CI remain the same without the extra feedback loop and the degradation curve remains completely linear. In the TES, the addition of the second positive feedback loop, which contains the protease, changes the protein degradation from linear (dotted-dash line) to nonlinear (dashed line) while the CI production curve (solid line) remains unchanged (Figure 1e). The rate-balance plots show two different paradigms for creating bistable switches. One paradigm adds nonlinearity to activator production (TS), while the other adds nonlinearity to the activator degradation (TES).

Conceptually, TES topologies have the benefit of a larger bistable parameter space, allowing for increased robustness and easier parameter tuning [12]. Furthermore, enzymatic reactions have faster response times compared to transcriptional interactions. Enzymatic reactions directly deactivate proteins, by dephosphorylation, proteolysis, etc. In TS topologies, protein removal is also required to flip state after the deactivation of a promoter; this usually occurs through dilution by cell division or targeted protein degradation (e.g. ssrA tags). Increasing the rate of protein degradation in TS topologies 
allows for faster switching speeds, however, this further limits the already narrow parameter space of TS topologies.

Synthetic TS have been dominant in prokaryotic systems. This is likely due to the higher modularity and orthogonality that transcriptional parts have relative to enzymatic parts. Interconnections between transcriptional devices (a transcription factor and its cognate promoter) are simply created by adjacent placement in the DNA sequence of a regulated promoter and a transcription factor from two transcriptional devices. This allows for virtually any transcriptional device to serve as an input or output to any other transcriptional device. This contrasts with creating interconnections to inputs and outputs of enzymatic steps, where a common molecule is required and generally this need for a shared molecule greatly limits the number of parts an enzyme can be connected to. Moreover, many of the characterized enzymes are responsible for global housekeeping or are involved in metabolic pathways, limiting the applicability of any prokaryotic TES system relying on these enzymes. Proteases that are targeted to proteins with specific terminal sequences are not as constrained as other enzymes with respect to their modularity, as this class of proteases are not restricted to a single substrate, but can be used with any protein that will retain activity with the addition of the protease targeting signal.

\section{Results}

\section{Cl-mf-ssrA can activate $\mathrm{P}_{\mathrm{RM}}$}

First, it is necessary to check that the addition of the $m f$ ssrA tag onto the C-terminus of $\mathrm{CI}$ does not interfere with activation of $\mathrm{P}_{R M}$ by $\mathrm{CI}$. The $\mathrm{N}$-terminal domain of $\mathrm{CI}$ contains a DNA binding domain, and the C-terminal domain includes a dimerization domain [36]. CI first dimerizes and then binds onto $\mathrm{O}_{R} 1$ and $\mathrm{O}_{\mathrm{R}} 2$ to activate $\mathrm{P}_{R M}$ and repress $\mathrm{P}_{R}$. Under normal physiological expression levels of CI the C-terminal domain is necessary for dimerization. Dimerization in turns helps with DNA binding. When using $\mathrm{CI}$ with a truncated C-terminal domain, higher concentrations of CI are necessary for dimerization to occur and thus higher CI concentration is necessary for DNA binding [37]. The addition of a degradation tag may interfere with proper protein interactions, and thus needs to be tested.

CI tagged with an E. coli ssrA tag (CI-ec-ssrA) has been shown to not interfere with native activity [38]. CI$e c$-ssrA also shows a shortened CI half-life [38,39]. Tagged CI has been widely used in various applications and retains activity $[38,40]$. We tested the ability of CI$m f$-ssrA, CI-ec-ssrA, and untagged CI (CI-wt) to active $\mathrm{P}_{R M}$ (Figure $2 \mathrm{a}$ and $\mathrm{b}$ ). In this test $\mathrm{CI}$ production was induced by IPTG, resulting in $\mathrm{P}_{R M}$ activation and GFP expression. All three variants of CI properly activated $\mathrm{P}_{R M}$. Across all IPTG concentrations, CI-wt had the

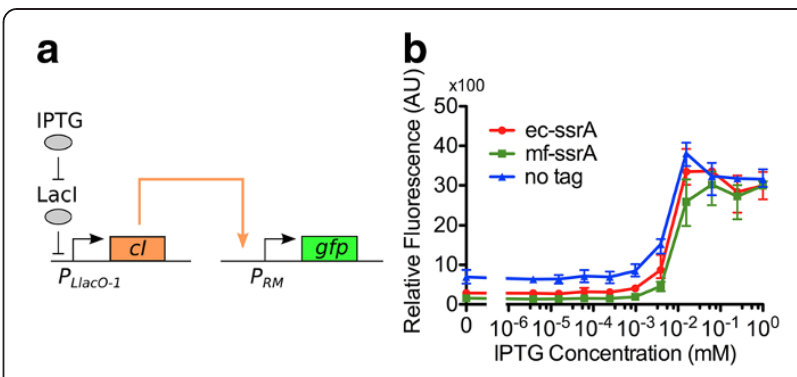

Figure $2 \mathbf{C l}$ turning on $\mathbf{P}_{\boldsymbol{R M}}$. (a) A two plasmid system was used to test the ability of $P_{R M}$ activation by $\mathrm{Cl}$. This circuit consists of an IPTG inducible plasmid that expresses $\mathrm{Cl}$ and a reporter plasmid expressing GFP by activation of $\mathrm{P}_{R M}$. Two different ssrA tags were added to the $\mathrm{Cl}$ protein (ec-ssrA, $m f$-ssrA) to test the effect of ssrA tags on $P_{R M}$ activation. (b) Fluorescence measurements were normalized to $\mathrm{OD}_{600}$ to account for cell density changes. Strains were cultured in various concentration of IPTG to test for $P_{R M}$ activation. Error bars denote the measurement range (highest and lowest value). All data points were taken with three biological replicates.

highest GFP expression and CI-mf-ssrA generally had the lowest GFP expression.

As stated above, the activity of $\mathrm{P}_{R M}$ is not monotonic with $\mathrm{CI}$ concentration; above a threshold concentration of $\mathrm{CI}$ the activity of $\mathrm{P}_{R M}$ decreases with increasing $\mathrm{CI}$. However, even for the $\mathrm{P}_{R M}$ promoter with mutated $\mathrm{O}_{\mathrm{R}} 3$ operator site, all three CI variants showed slight repression of $\mathrm{P}_{R M}$ at high levels of CI. CI-wt showed the highest degree of repression.

These results show the ability for CI- $m f$-ssrA to activate $\mathrm{P}_{R M}$, and thereby CI- $m f$-ssrA is usable for the proposed the TES. The results also show CI- $m f$-ssrA behavior slightly deviates from wild-type function; this will be discussed below.

\section{Cl-mf-ssrA can be degraded by mf-Lon}

Orthogonality between the M. florum and E. coli ssrA systems has been demonstrated [25]. Given this, we investigated the ability of $m f$-Lon to degrade CI- $m f$-ssrA. We also needed to check for specificity between $m f$-Lon and its cognate tag ( $m f$-ssrA). We tested the ability for $m f$-Lon to shut off the $\mathrm{P}_{R M^{-}} \mathrm{CI}$ positive feedback loop (Figure 3a). We used the plasmids PRM-CIwt-TetR, PRM-CILVA-TetR, and PRM-CImf-TetR (Table 1) for this test; each plasmid contained a different CI/ssrA tag condition. This test circuit is almost the complete TES bistable network. The $m f$-Lon/TetR loop is open, so this test circuit does not form a bistable circuit. Instead, $m f$ Lon production was induced by IPTG, so $m f$-Lon production should be unaffected by both TetR and aTc concentrations. We utilized both GFP reporter plasmids with our test circuit. The PRM-GFP reporter plasmid more directly tested the $\mathrm{P}_{R M^{-}} \mathrm{CI}$ loop state. The PLtetO1-GFP plasmid reported on the TetR production level. 

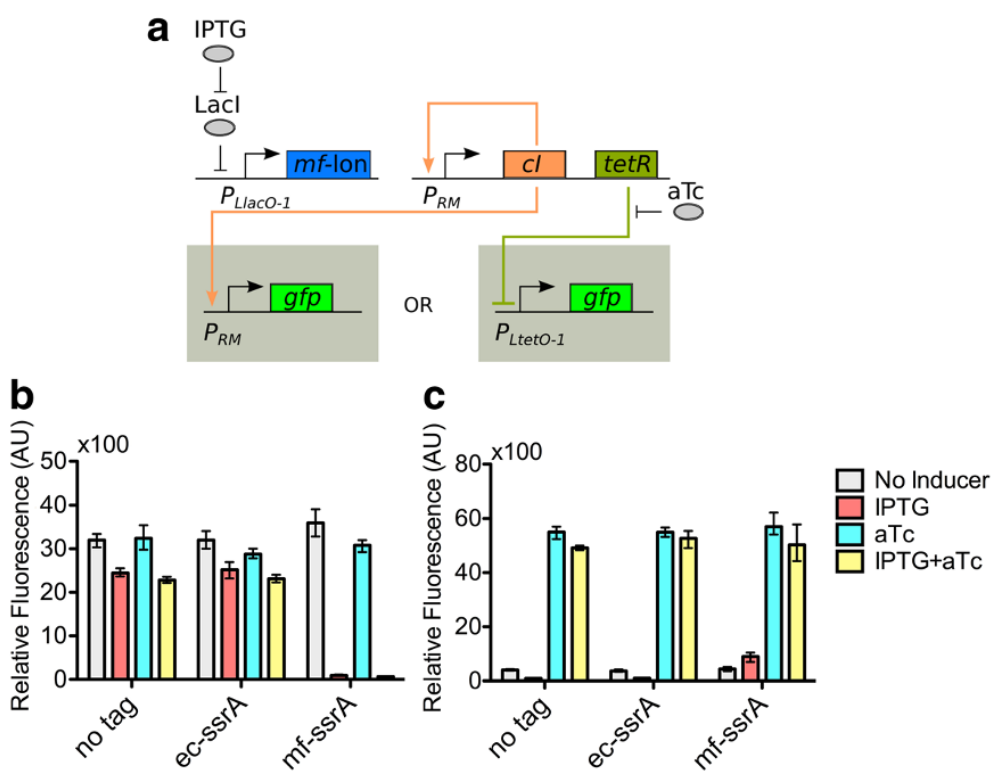

Figure 3 Testing the $\boldsymbol{m f}$-Lon protease. (a) A three plasmid system was used to test $\mathrm{Cl}$ degradation by $m f$-Lon. The circuit has a positive feedback loop consisting of $\mathrm{P}_{R M}$ expressing its activator, Cl. Another plasmid expressed $m$ f-Lon when induced with IPTG. Three different ssrA tag conditions were used for the activator (ec-ssrA, $m f-s s r A$, no tag). When $m f$-Lon is expressed a properly tagged $\mathrm{Cl}$ should degrade, thus turning off the positive feedback loop. (b) The PRM-GFP reporter was used to measure the presence of Cl. (c) ThePLtetO-1-GFP reporter was used to test the presence of TetR. All data points were measured using three biological replicates. Error bars indicate data range (highest and lowest value).

The $\mathrm{P}_{R M^{-}} \mathrm{CI}$ positive feedback loop was functional using all three variants of CI (Figure 3b). All three CI variants, in the positive feedback loop configuration, showed strong levels of GFP expression (reporting with PRM-GFP). Expression of $m f$-Lon was only able to degrade CI- $m f$-ssrA and thus turn "off" the $\mathrm{P}_{R M^{-}} \mathrm{CI}$ loop, resulting in greatly decreased GFP production. CI-wt and CI-ec-ssrA could not be degraded by $m f$-Lon and the GFP production did not change as drastically. This shows $m f$-Lon specificity to its cognate $s s r A$ tag. In both the CI-wt and CI-ec-ssrA circuits, when IPTG addition was added, the $\mathrm{P}_{R M}$-CI loop remained "on," however there was a measurable decrease in GFP expression. The decrease in GFP expression can probably be attributed to the increased metabolic burden from the expression of $m f$-Lon on a plasmid with a ColE1 origin of replication.

The PLtetO-1-GFP reporter tested TetR production level. TetR is biscistronically expressed with $\mathrm{CI}$ and the concentration of TetR should be approximately proportional to $\mathrm{CI}$ concentrations. When using the PLtetO-1GFP reporter, GFP expression increased only in the PRM-CImf-TetR test circuit (Figure 3c). This result again confirmed the specificity of $m f$-Lon to the $m f$-ssrA tag. GFP expression decreased with $m f$-Lon induction from test circuits expressing CI-wt and CI-ecssrA (Figure 3c). Once again, the decrease in GFP expression is likely due to increased metabolic load from $m f$-Lon induction.
The addition of aTc showed high GFP expression levels in all three experimental strains containing the three variants of CI (reporting with PLtetO-1-GFP). The addition of aTc inactivates TetR and $P_{\text {LtetO-I }}$ becomes fully active regardless of $m f$-Lon or CI expression. Even when the $\mathrm{P}_{\mathrm{RM}}$-CI positive feedback loop was shut "off" (resulting from CI- $m f$-ssrA degradation by $m f$-Lon), there was always basal TetR expression, which kept $\mathrm{P}_{\text {LtetO-1 }}$ partially off. Only when aTc was added would $\mathrm{P}_{\text {LtetO-1 }}$ become fully active. It is important to keep in mind that in these test circuits the TetR expression level is always high enough to have measurable effect on $\mathrm{P}_{\text {LtetO-1 }}$ regardless of the $\mathrm{P}_{R M^{-}}$CI state.

From these experiments we concluded that CI-mf-ssrA could indeed be degraded by $m f$-Lon. Just as important, $m f$-Lon specifically degrades proteins tagged with $m f$ ssrA and does not degrade proteins tagged with $e c$-ssrA.

\section{Creating a 5'-UTR library for mf-Lon}

We created a library of 5'-UTRs, which gave a range of $m f$-Lon expression levels. Initially the RBS calculator was used to create a 5 ' untranslated region (5'-UTR) for the $m f-$ lon gene [41]. Two different schemes were used to mutate the 5'-UTRs (Figure 4a). The first method randomized the 6 base pairs before and 6 base pairs after the Shine-Dalgarno (SD) region. The second method randomized three base pairs within the $\mathrm{SD}$ consensus region. To quantify the relative expression levels from the 5'-UTRs in the relevant context, these 5'-UTRs were 


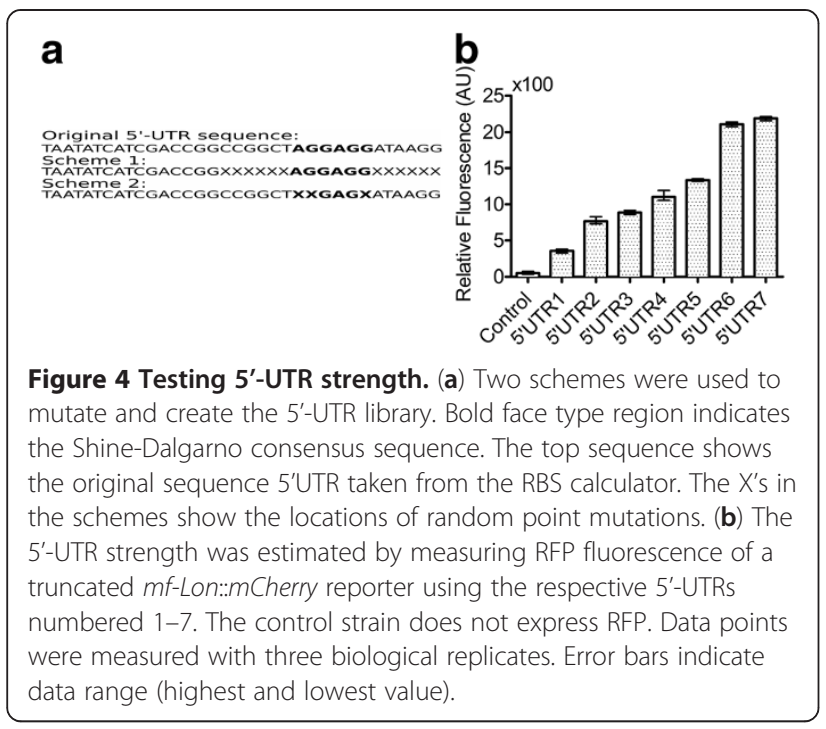

added on a truncated $m f$-lon fused with mCherry [42]. This fusion protein was constitutively expressed using the $\mathrm{P}_{\text {LtetO- } 1}$ promoter. Relative expression strength levels were assumed to be proportional to the measured RFP fluorescence of the fusion protein. A total of 192 colonies were screened for their level of RFP expression. Seven 5'-UTRs with different RFP expression levels (Figure 4b) spanning the complete RFP expression range of the library were selected and sequenced (Table 2). These seven 5'-UTRs were then incorporated into the switch construct. The 5'-UTRs were numbered 1-7 with 5'UTR1 being the weakest 5'-UTR and 5'UTR7 being the strongest 5'-UTR. The switches incorporating the respective 5'-UTR were named in the same fashion (e.g. SW1 refers to a switch which incorporates 5'UTR1).

\section{Tuning mf-Lon production with RBS strength yields bistable switches}

The presence or absence of bistable behavior was determined by measuring hysteresis. SW1, SW2 and SW3 were assayed along using the PRM-GFP reporter plasmid and showed "always on" behavior, indicating $m f$-Lon expression was too low to turn off the $\mathrm{P}_{R M^{-}} \mathrm{CI}$ loop.

\section{Table 2 5'UTR sequences}

\begin{tabular}{ll}
\hline Name & Sequence \\
\hline Original & TAATATCATCGACCGGCCGGCTAGGAGGATAAGG \\
\hline 5'UTR1 $^{\prime}$ TAATATCATCGACCGGCCGGCTCCGAGCATAAGG \\
\hline $5^{\prime}$ UTR2 & TAATATCATCGACCGGCCGGCTCAGAGCATAAGG \\
\hline $5^{\prime}$ UTR3 & TAATATCATCGACCGGCCGGCTAAGATAAGG \\
\hline 5'UTR4 $^{\prime}$ TAATATCATCGACCGGCCGGCTGAGAGAATAAGG \\
\hline $5^{\prime}$ UTR5 & TAATATCATCGACCGGCCGGCTACGAGGATAAGG \\
\hline $5^{\prime}$ UTR6 & TAATATCATCGACCGGCCGGCTAGGAGGATAAGG \\
\hline $5^{\prime}$ UTR7 & TAATATCATCGACCGGAGAAGAAGGAGGTGCTGGT \\
\hline
\end{tabular}

Even with full TetR inactivation using saturating levels of aTc, the $\mathrm{P}_{R M^{-}}$CI loop remained "on." No hysteresis was measured in SW1, SW2 and SW3. The behavior of SW3 (Figure 5a) is representative of SW1, SW2 and SW3.SW4, SW,5 and SW6 with PRM-GFP displayed bistable behavior. The behavior of SW4 (Figure 5b) is representative of SW4, SW,5 and SW6. For SW4, SW,5 and SW6 we observed a bistable region at low levels of inducers $(0-0.4 \mathrm{ng} / \mathrm{mL}$ aTc and $0-0.016 \mathrm{mM}$ IPTG). At high levels of inducer we observed monostability where the two curves converged. Table 3 summarizes the behavior of SW1 through SW7.

SW7 was assayed using with the PRM-GFP reporter plasmid and showed monostable behavior; the switch was always off unless high levels of IPTG were used to induce high levels of $\mathrm{CI}$ production (Figure $5 \mathrm{c}$ ).

Hysteresis was not observed for any of the switches when using the PLtetO-1-GFP reporter (Figure 6). This however does not indicate the lack of bistable behavior

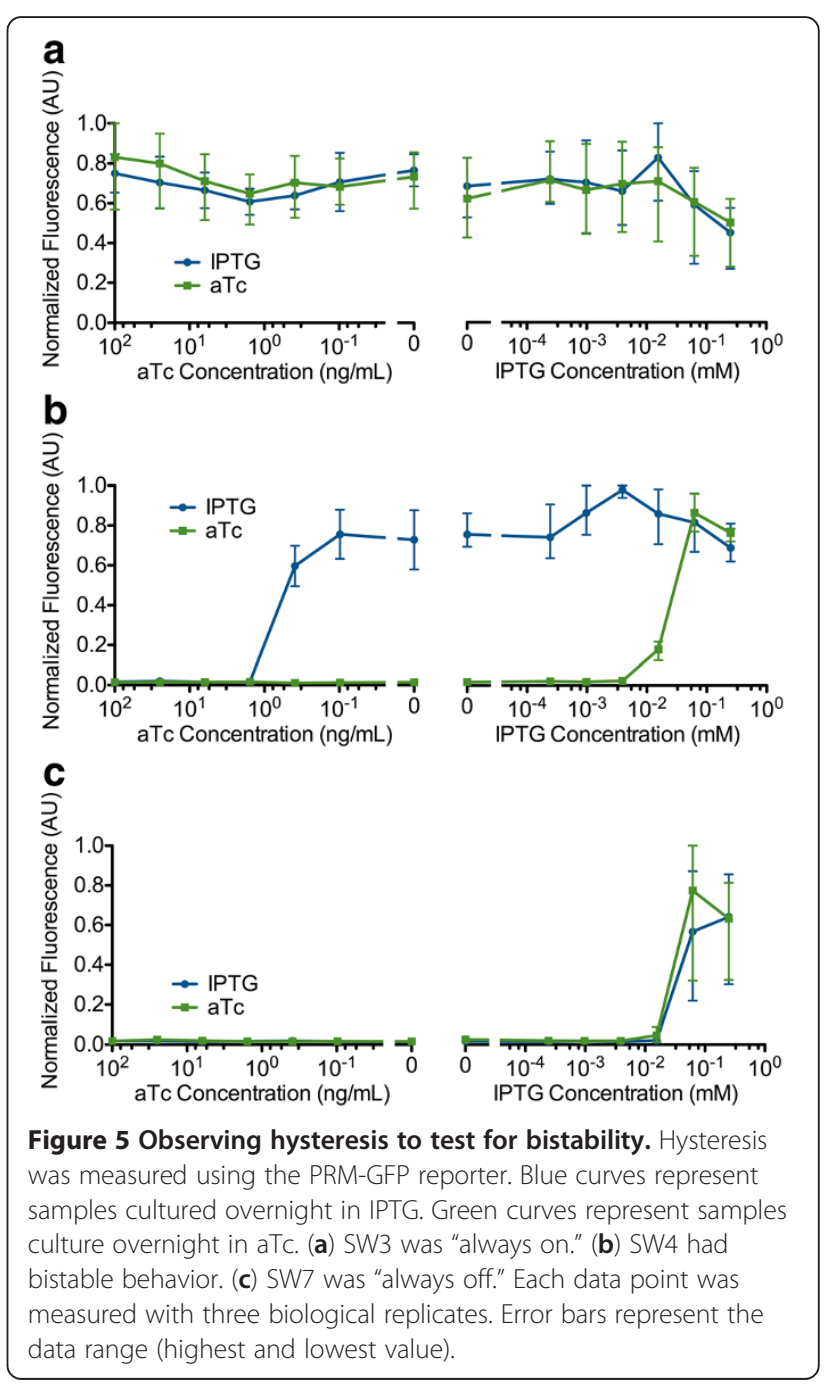


Table 3 Switch behavior (in the absence of inducers)

\begin{tabular}{ll}
\hline Name & Behavior \\
\hline SW1 & Monostable "on" \\
\hline SW3 & Monostable "on" \\
\hline SW4 & Monostable "on" \\
\hline SW5 & Bistable \\
\hline SW6 & Bistable \\
\hline SW7 & Bistable \\
\hline
\end{tabular}

in SW4, SW5 and SW6. The lack of hysteresis observed using the PLtetO-1-GFP reporter is discussed later.

Both states of a tuned switch are stable over 40 doublings

A long-term stability test was performed on SW4 to check for the stability of both states. The strains were able to hold their states throughout the 32 hour period ( $\sim 40$ cell divisions) (Figure 7$)$.

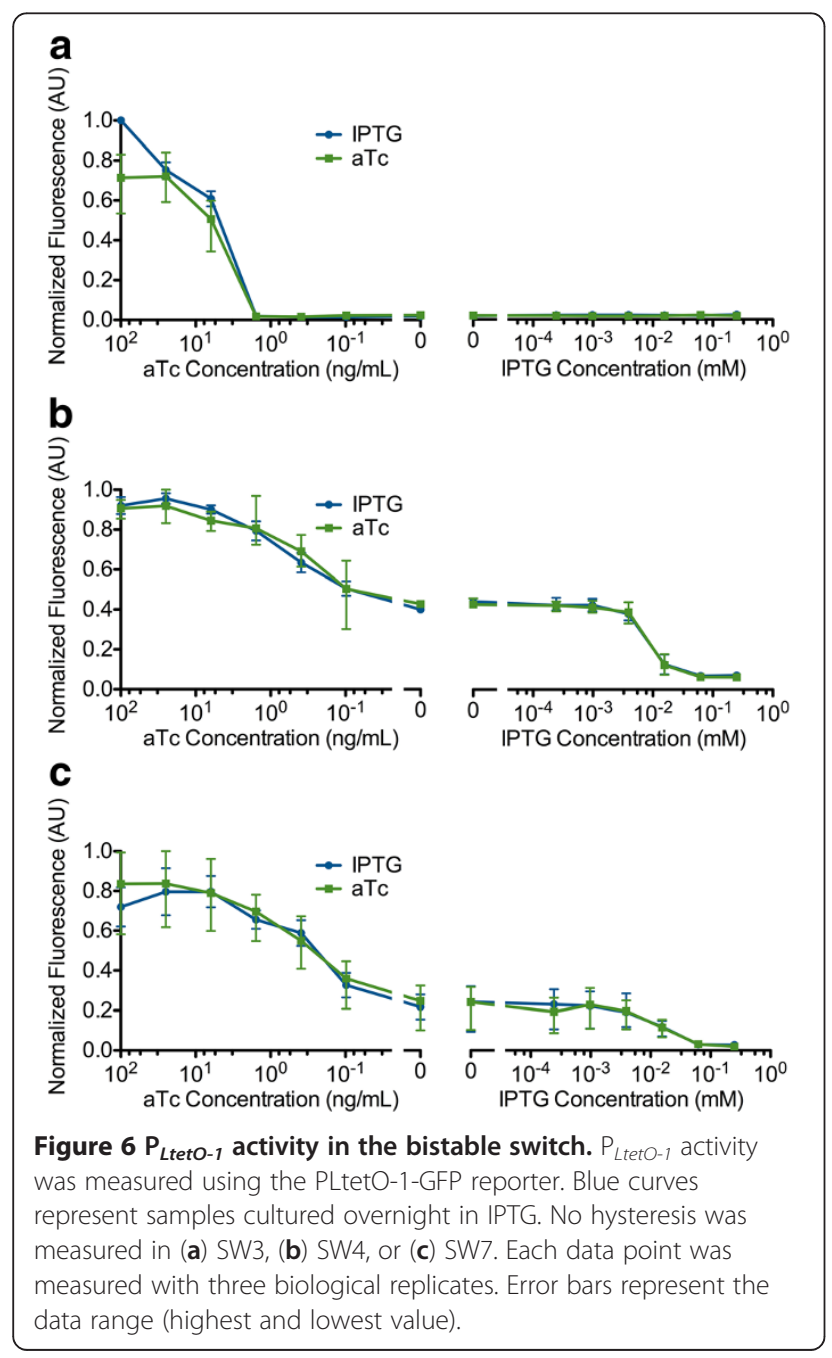

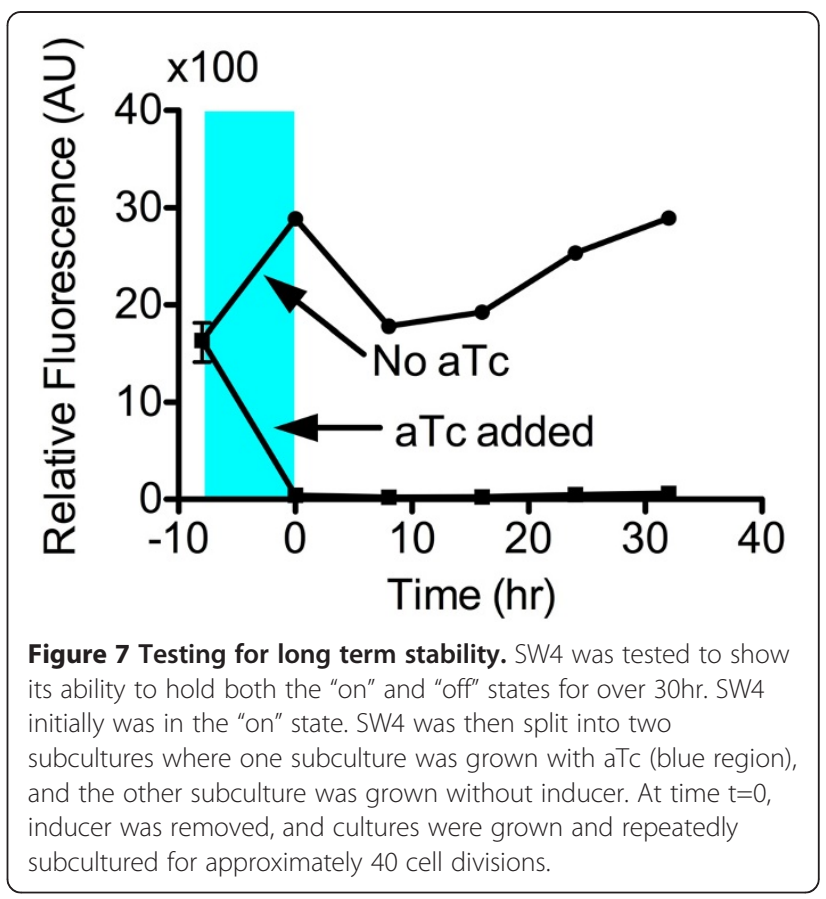

\section{Transient behavior of switches}

We tested the transient behavior of SW4, SW5 and SW6. Both GFP reporters were used in this set of experiments. We tested for activation time and deactivation time using the PRM-GFP reporter. Activation time $\left(\mathrm{ACT}^{1} \frac{1}{2}\right)$ was defined as the time necessary for GFP expression to go from the initial low expression to $50 \%$ expression. Deactivation time (DCT $1 / 2$ ) was defined as the time necessary for GFP expression to decrease to $50 \%$ expression. Each strain was initially forced into either the "on" or "off" state. Next, inducers were removed and strains were allowed to reach steady-state. The appropriate inducer was then added to change states. We also measured the behavior when no inducer was added in order to observe transient state persistence. Lastly, we also measured transient behavior when inducer was added to reinforce the state (e.g. adding aTc to a switch already in the "off" state). Only results for SW4 are shown (Figure 8). Results for SW5 and SW6 are shown in Additional file 1: S.2.

When no inducer was added, each strain was able to hold its previous state. When inducer was added to reinforce the state, each strain kept its previous state. Both the "reinforcement" and "no inducer" curves were similar. When inducer was added to change the state, we quantified the ACT $1 \frac{1}{2}$ and $\mathrm{DCT}^{1 / 2}$ (Table 4). $\mathrm{ACT}^{1 / 2}$ was similar for SW4, SW5 and SW6 with all three switches reaching $50 \%$ activation in approximately 1 hour. DCT $1 / 2$ was as expected, with SW4 having the longest $\mathrm{DCT}^{1 / 2}$ (139 $\left.\mathrm{min}\right)$ and SW6 having the shortest (83 min). 


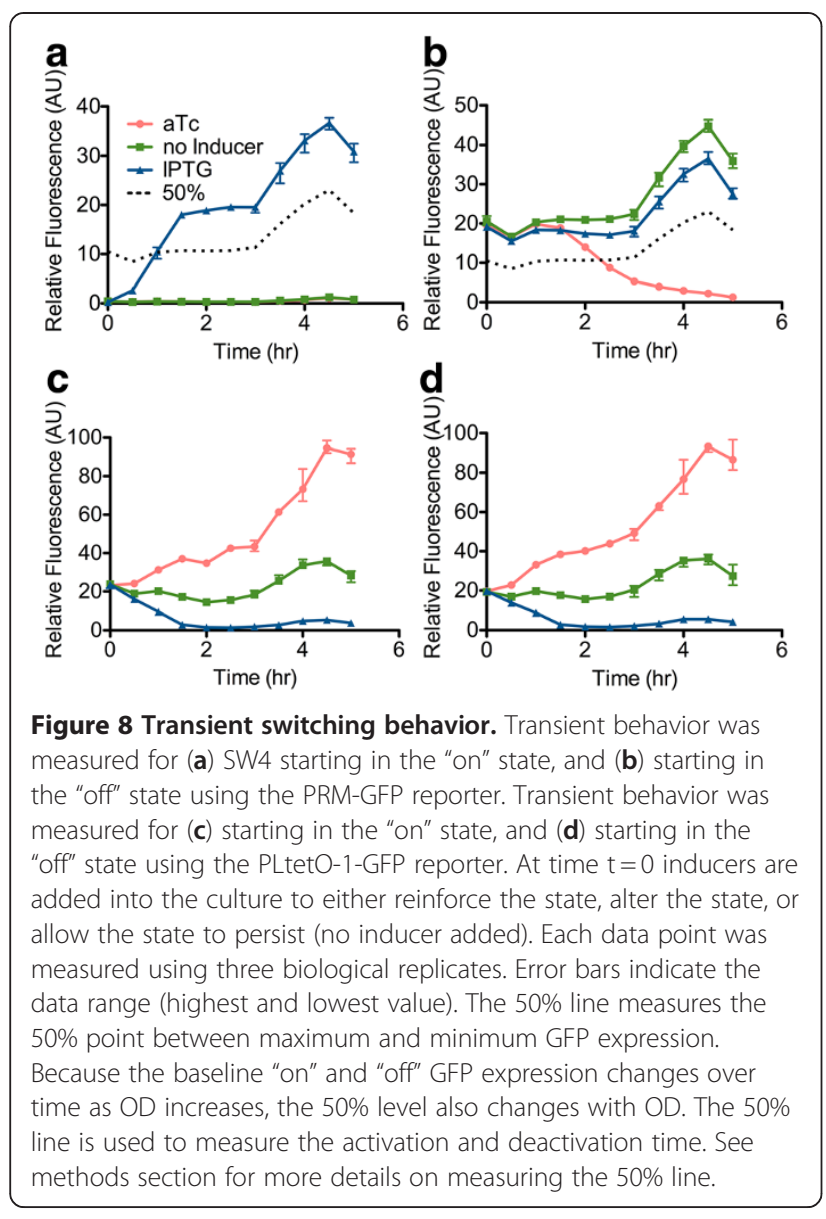

Activation was dependent on $\mathrm{CI}$ accumulation rate. To first order, the $\mathrm{CI}$ accumulation rate is dependent on $\mathrm{CI}$ production rate. All strains had the same inducible CI plasmid, and identical $\mathrm{P}_{R M^{-}}$CI positive feedback loops. As a result, all three switches should have the same CI production rates. As observed, $\mathrm{ACT}^{1 / 2}$ for all three strains did not differ by much (as compared to DCT $1 / 2$ ). Protein degradation rate is dependent on protease concentration, giving the expected results for deactivation time. Once again when we reported SW4, SW5 and SW6 with PLtetO-1-GFP (Figure 8c and d), no hysteresis was observed. The curves in the "on" state or "off" state are identical.

Given the fast deactivation of SW6, the dynamic measurements of SW6 were repeated using flow cytometry

Table 4 Activation deactivation times for 3 biological replicates

\begin{tabular}{|c|c|c|c|c|}
\hline Name & $\begin{array}{l}\text { Average } \\
\mathrm{ACT} 1 / 2\end{array}$ & ACT $1 \frac{1}{2}$ Range & $\begin{array}{l}\text { Average } \\
\text { DCT } 1 \% 2\end{array}$ & DCT $1 \frac{1}{2}$ Range \\
\hline SW4 & $59 \min$ & 55-64 min & $139 \mathrm{~min}$ & $137-140 \mathrm{~min}$ \\
\hline SW5 & $53 \mathrm{~min}$ & 48-57 min & $90 \mathrm{~min}$ & 82-98 min \\
\hline SW6 & $65 \mathrm{~min}$ & $61-67 \mathrm{~min}$ & $83 \mathrm{~min}$ & $81-85 \mathrm{~min}$ \\
\hline
\end{tabular}

(Supplemental Information S.3.1) to ensure that the "on" state and "off" state were each composed of a unimodal distribution of cells in that state. The flow cytometry data confirms that each state contains a unimodal distribution of cells.

\section{Discussion}

We present a new bistable topology in E. coli. Our system specifically uses an ssrA degradation system orthogonal to the native $E$. coli system. The usage of the $m f$-Lon protease and $m f$-ssrA tag in a useful circuit had not previously been explored. The usage of nonlinear protein degradation in a bistable switch has mainly been a theoretical discussion and has not been synthetically employed. In this paper we experimentally tested the effect of using nonlinear protein degradation in a synthetic prokaryotic system.

\section{Cl-mf-ssrA degradation}

We found that CI-wt activates $\mathrm{P}_{R M}$ the most strongly as compared to CI with ssrA tags (Figure $2 \mathrm{~b}$ ). It is known that the CI dimer is extremely stable and has a long half-life [17]; even with low expression rates CI-wt can quickly accumulate and activate $\mathrm{P}_{R M}$. The $m f$-ssrA tag should be completely orthogonal to the ec-ssrA tag [25]. In $E$. coli, the half-life of proteins tagged with $m f$-ssrA should be unaffected since they will not be targeted for degradation. We report that CI-mf-ssrA has a different behavior than CI-wt. CI- $m f$-ssrA requires a higher expression rate to activate $\mathrm{P}_{R M}$ compared to CI-wt. It requires a higher expression rate than even $\mathrm{CI}-e c$-ssrA. This implies that CI-mf-ssrA is intrinsically unstable and degrades quickly, even when it is not targeted for degradation. Most likely the addition of 27 aa ssrA tag interferes with the $\mathrm{C}$-terminal domain of the lambda repressor, as opposed to the 13 aa $e c$-ssrA tag. The $m f$ ssrA tag disrupts the C-terminal domain dimerization of $\mathrm{CI}$, requiring a higher concentration of $\mathrm{CI}$ for dimerization. Furthermore, CI dimers are cooperatively stabilized. Unless there are high expression levels of CI, few $\mathrm{CI}$ dimers are formed and the unstable $\mathrm{CI}$ monomers are quickly degraded [17]. Since $m f$-ssrA can interfere with protein functionality, this implies that the number of proteins that can be used with the $m f$-ssrA degradation system may be limited.

\section{Controlling switching speeds by dynamically expressing a protease}

Enzyme substrate reaction rates are dependent on both the concentration of the enzyme and the substrate. The maximum reaction rate, $\mathrm{V}_{\max }$, is directly proportional to enzyme concentrations. If switching speeds need to be modified, adjusting protease expression levels provides an easy way to tune the switching speed. In our reported 
TES, changing protease expression levels only changed the deactivation speed. The activation speed is most likely controlled by changing the $\mathrm{CI}$ expression rate on the PLlacO-1-CImf plasmid (this was not explicitly tested).

It should be noted that on TS, changing the expression levels of transcriptional activators and repressors does not change the activation and deactivation speeds significantly, because protein degradation is the limiting factor and is unchanged by protein expression rates. The addition of degradation tags in a TS may not be an option due to parameter constraints. In our TES, protein degradation is not a limiting factor, because proteins are either tagged with a fast degradation tag or dynamically degraded by a protease, which allows for flexibility in changing activation and deactivation speeds.

The TES topology presented can be rendered modular by using two input promoters. One promoter would express $\mathrm{CI}$ and the other would express $m f$-Lon. In this scheme $\mathrm{DCT}^{1 / 2} / 2$ would depend on both the expression rate of $m f$-Lon from the linking circuit as well as the expression rate on the switch itself. Depending on relative rates within the switch and on the input, the faster rate would dominate the other. The switching "on" rate would be dominated by $\mathrm{CI}$ production rate on the input circuit.

\section{The lack of hysteresis from $\mathrm{P}_{\text {LtetO-1 }}$}

Both the hysteresis data and transient behavior data confirmed the lack of hysteresis from $\mathrm{P}_{\text {LtetO-1 }}$. When using the PLtetO-1-GFP reporter, flow cytometry experiments showed that in the absence of inducers, cells in either the "on" or "off" state had identical GFP expression levels (Additional file 1: S.3.3). With the addition of aTc we observed high levels of GFP expression in a tightly distributed unimodal population (Additional file 1 : S.3.2). When IPTG was added, there appeared to be a bimodal population of cells; one population did not express GFP, the other population expressed low levels of GFP. The bimodal population of cells implied that TetR did not completely repress $\mathrm{P}_{\text {LtetO-1 }}$. However, TetR production was clearly high enough to prevent a fully active $\mathrm{P}_{\text {LtetO-1 }}$ promoter. We saw that even when the switch was "off," there was enough TetR expression to keep $\mathrm{P}_{\text {LtetO-1 }}$ partially off and only with the addition of aTc would $P_{\text {LtetO-1 }}$ be fully active. It is unknown why the addition of IPTG was unable to completely shut off $\mathrm{P}_{\text {LtetO-1 }}$.

Implications regarding the lack of hysteresis from $\mathrm{P}_{\text {Lteto-1 }}$ One last observation is that the observed lack of hysteresis in $m f$-Lon concentration may make it unnecessary to add a second positive feedback loop to create a bistable network. Instead, bistable behavior may be possible using only a single positive feedback loop along with constitutive expression of $m f$-Lon. The simplified circuit would consist of a $\mathrm{P}_{R M^{-}}$-CI positive feedback back loop, with CI tagged with $m f$-ssrA. The expression level of $m f$-Lon would determine the degradation rate of CI$m f$-ssrA (Supplemental Information S.4.3). Using the literature values of the reported Michaelis constant for $m f$-Lon $\left(K_{\mathrm{m}}\right)[25]$ and the literature value for the dissociation constant of CI-wt to $\mathrm{P}_{R M}$ binding $\left(K_{\mathrm{dCI}}\right)$ [33], bistable behavior cannot be obtained with a single positive feedback loop. Using modified $K_{\mathrm{m}}$ where $K_{d C I}>K_{m}$, bistability is analytically observed using a rate balance plot (Additional file 1: S.4.4).

The literature value for $K_{m}$ may be inaccurate, since $K_{\mathrm{m}}$ was measured in vitro. The in vivo value may actually be lower value than the measured in vitro $K_{m}$ value. The native function of $m f$-Lon is to rescue stalled transcription events. With a high $K_{m}$, stalled events cannot be rescued efficiently. Natively, $m f$-Lon is a housekeeping gene, and expression of the protease is probably low. The in vivo functioning of $m f$-Lon is most likely fast and efficient, even at low protease concentrations. In order to create the bistable rate-balance plot, $K_{m}$ needs to be decreased by three orders of magnitude, and this shift in value is also highly doubtful. The lack of hysteresis for $P_{\text {LtetO- } 1}$ and its implications require more tests to be fully understood.

\section{Comparison of a degradative a TES vs the tradition TS}

The principal benefit of the protein degradative interaction in the TES is the tunability of switching speeds due to active removal of proteins through a protease. In the TS, removal of proteins is through dilution by cell division. The original toggle [14] switch required 34 hrs for to begin switching, and over 5 hours for the switch to completely change states. Switching states in the linked double positive feedback loop reported by Chang et al. [10] required the dilution of both proteins and the small molecule IPTG. In this case switching required a 1 million-fold cell dilution for proper switching. With active protein removal through a protease we were able to complete switching in approximately 3 cell divisions $(\sim 2.5 \mathrm{hr})$. In theory, switching could be accomplished within one cell division. This would require the simultaneous tuning of the RBS for all three proteins (CI, TetR and $m f$-Lon) to optimize deactivation time.

Another benefit of our TES compared to a TS is the increased robustness of the TES. It has been previously shown that these types of hybrid circuits are more robust than transcription-only circuits [12]. Adding a protease introduces nonlinear degradation and thereby adds nonlinearity into the system. In classic TS systems, some nonlinearity can be added by increasing the apparent cooperativity in the main positive feedback loop. Additionally, it is difficult to add ec-ssrA tags in the TS, 
since the parameter space is already very small. Shortening the protein lifetime also decreases the robustness of the circuit. In the TES, the parameter space was broad enough to allow for the addition of ec-ssrA tags. It can be argued that increased bistable parameter space contributes to the tunability of switching time.

\section{Conclusions}

We have demonstrated a hybrid bistable switch using both transcriptional components and an enzyme. Bistable behavior is achieved through nonlinear degradation of the transcriptional activator. A dynamically expressed ssrA protease orthogonal to the $E$. coli system was used in order to achieve nonlinear protein degradation. Use of a protease allows for an easy way to tune switching speed in bistable circuits. This is a new method of creating synthetic bistable networks, which have been theoretically proposed, but have not been tried experimentally.

\section{Materials and Methods}

Bacterial strains, media, and growth conditions

Cloning steps and experiments were performed with E. coli strain DH10B. Overnight cultures were performed in Luria-Bertani (LB) medium at $37^{\circ} \mathrm{C}$. All experiments were performed in EZ-rich media with $1 \%$ glucose, purchased from Teknova and were grown at $37^{\circ} \mathrm{C}$. Antibiotics were used with the following concentrations: kanamycin at $40 \mu \mathrm{g} / \mathrm{mL}$, chloramphenicol at $20 \mu \mathrm{g} / \mathrm{mL}$, ampicillin at $100 \mu \mathrm{g} / \mathrm{mL}$, carbenicillin at $100 \mu \mathrm{g} / \mathrm{mL}$. Experiments used carbenicillin in lieu of ampicillin. Cloning steps used ampicillin instead of carbenicillin. Restriction enzymes were purchased from Fermentas Inc. Polymerases were purchased from Finnzymes and Stratagene. Media, antibiotics and enzymes were used according to manufacturer recommendations.

\section{Plasmid construction}

Table 1 lists all the plasmids used in this study. All cloning plasmids in this study have the BglII/BamHI BglBrick sites [43]. Ribosome binding sites (RBS) were added using 'Round-the-horn site directed mutagenesis $(\mathrm{RTH})$ and its respective primers [44]. RBS were designed using the RBS calculator. IPTG inducible plasmids with $\mathrm{Cm}$ marker were constructed by inserting the respective genes into BglBrick sites using the XhoI and BglII restriction sites. $M f$-ssrA and $e c$-ssrA tags (AANDENYALVA) [45] were added using RTH and its respective primers. CI-ec-ssrA, sfGFP, TetR and $m f$-Lon were destabilized with the addition of a ec-ssr $A$ tag.

Mf-lon was obtained from Addgene (Plasmid 21867: pBAD33-mf-lon) [25]. The sequence was amplified with PCR adding BglBrick sites then added into a high copy cloning plasmid with pUC19 origin of replication and ampicillin marker. Superfolder GFP (sfGFP) is the same as described by Pedelacq et al. [31]. The RFP, mCherry, is described by Shaner et al. [42].

\section{Florescence measurements for endpoint measurements}

Endpoint measurements started with a $5 \mathrm{~mL}$ LB overnight culturing with $200 \mathrm{rpm}$ of continuous shaking. After overnight culturing each sample was subcultured with a 100 fold dilution into $5 \mathrm{~mL}$ of EZ-rich media and regrown to an $\mathrm{OD}_{600}$ of 0.4 . The cultures were then subcultured into EZ-rich media to an OD of 0.05 with added inducers. Florescence measurements were measured by transferring $150 \mu \mathrm{L}$ of culture into a 96 well microplate to measure $\mathrm{OD}_{600}$ and fluorescence on a Molecular Devices Spectramax M2. GFP measurements used $485 \mathrm{~nm}$ for excitation and $538 \mathrm{~nm}$ for emission. RFP measurements used $584 \mathrm{~nm}$ for excitation and $612 \mathrm{~nm}$ for emission. All fluorescence measurements were normalized to $\mathrm{OD}_{600}$. All reported measurements were repeated in triplicate by using three different colonies.

\section{Hysteresis measurements}

A single colony was picked out a plate transformed with the relevant plasmids and cultured overnight in both $5 \mathrm{~mL} \mathrm{LB}$ with IPTG $(.0625 \mathrm{mM})$ and $5 \mathrm{~mL} \mathrm{LB}$ with saturating aTc $(100 \mathrm{ng} / \mathrm{mL})$ to force them into the high and low state respectively. Generally $1 \mathrm{mM}$ of IPTG was used for saturating conditions. We found that reduced levels of IPTG were sufficient to induce high levels of CI and push switches into the "on" state. The reduced levels of IPTG also allowed for easier removal of IPTG. The overnight cultures were washed three times with EZ-rich media and split into multiple subcultures on a 96 deep well plate. Each subculture was grown with a total of $1 \mathrm{~mL}$ of media and the plate was shaken at $250 \mathrm{rpm}$. More specifically each overnight sample was subcultured into 7 different IPTG conditions (ranging from $0 \mathrm{mM}$ to $.25 \mathrm{mM}$ ) and 7 different aTc conditions (ranging from $0 \mathrm{ng} / \mathrm{mL}$ to $100 \mathrm{ng} / \mathrm{mL})$. After cell growth reached mid$\log (\sim 8 \mathrm{hrs})$, each sample on the 96 well plate was recultured on a new 96 well plate with fresh media using exactly the same inducer concentrations as the previous 96 well plate. This step ensured complete removal of the initial inducer. After the samples reached mid-log again, GFP output was measured. For each switch, GFP output was measured for both PLtetO-1-GFP and PRM-GFP reporters. Each data point was replicated three times using three individual bacterial colonies.

\section{Long term stability experiment}

The long term stability test was performed only on SW4 with the PRM-GFP reporter. SW4 was grown in $5 \mathrm{~mL}$ LB overnight with saturating IPTG $(.0625 \mathrm{mM})$. The overnight culture was washed three times and recultured in fresh EZ-medium using a 1000 fold dilution. After an 
$\mathrm{OD}_{600}$ of 0.5 was reached the culture was then split into two subcultures. Both were cultured with 1000 fold dilutions. One culture had $100 \mathrm{ng} / \mathrm{mL}$ of aTc added in order to switch in to the "off" state. The other culture was grown without aTc. After $8 \mathrm{hr}$ of growth, both samples were washed and subcultured in fresh media without any inducer, again with a 1000 fold dilution. Both samples were grown an additional $32 \mathrm{hr}$, washing and subculturing every $8 \mathrm{hr}$, again using 1000 fold dilutions. GFP measurements are taken every eight hours before each subculturing event. The doubling time was approximately 45 minutes, giving approximately 10 doubling every $8 \mathrm{hr}$. The total experiment had 40 doublings throughout the stability measurement. Each data point was repeated three times using three separate colonies.

\section{Transient switching measurements}

Transient measurements started with overnight cultures in $5 \mathrm{~mL}$ LB. One colony was picked from an agar plate to inoculate two $5 \mathrm{~mL} \mathrm{LB}$ culture tubes. One culture tube had $100 \mathrm{ng} / \mathrm{mL}$ of aTc, and the other tube had $0.0625 \mathrm{mM}$ of IPTG. After overnight growth (16 hr), the overnight cultures were washed three times in EZ-rich media and subcultured with 1000 fold dilution in EZrich media and regrown to an $\mathrm{OD}_{600}$ of approximately 0.8 . Each culture was split into three separate subcultures each with a different inducer $(0.25 \mathrm{mM}$ IPTG, $100 \mathrm{ng} / \mathrm{mL}$ aTc, and no inducer) and diluted down to an $\mathrm{OD}_{600}$ of 0.05 . The three different inducer conditions represent three transient conditions (change, reinforce, and persist). "Change" is when an inducer is added to alter the state. "Reinforce" is when the inducer used is the same as the overnight inducer. "Persist" is when no inducer is added and switches are allowed to keep their prior state. Fluorescence measurements were taken every 30 minutes. Measurements were all performed in triplicate using three separate colonies. GFP expression was reported using both reporters.

To measure $50 \%$ activation (ACT $1 / 2)$, we measured the time it took for a strain, initially in the "off" state, to reach $50 \%$ of maximum GFP expression. Because the baseline "on" and "off" GFP expression changes over time as OD increases, the 50\% level also changes with OD. The $50 \%$ level is found by taking the average value between the "on persist state" and "off persist state" for each time point. The $50 \%$ deactivation $\left(\mathrm{DCT}^{1 / 2}\right)$ level is found in a similar fashion as the $50 \%$ activation calculation. $\mathrm{ACT}^{1 / 2}$ and $\mathrm{DCT}^{1 / 2}$ were only measured using PRM-GFP. ACT $1 \frac{1}{2}$ and $\mathrm{DCT}^{1 / 2}$ were measured three times using three separate colonies.

\section{Flow cytometry}

Cultures to be measured were diluted 1:2000 in phosphate buffered saline (from Teknova) with $50 \mu \mathrm{g} / \mathrm{mL}$ of streptomycin. $150 \mathrm{~mL}$ of the diluted sample was placed in a 96 well microplate, and then measured on a Milipore guava easyCyte HT. For each sample approximately 2000 events were counted.

\section{Additional file}

Additional file 1: S.1 Comparison of TS and TES circuits [46-51].

\section{Competing interests}

The authors declare that they have no competing interests.

\section{Author contributions}

$\mathrm{DH}$ and $\mathrm{WH}$ conceived of the experiments. DH performed the experiments. $\mathrm{DH}, \mathrm{WH}$ and $\mathrm{MM}$ wrote the manuscript. All authors read and approved the final manuscript.

\section{Acknowledgements}

We would like to thank Justin Hsia and Murat Arcak for help with the analytical modeling and for comments on the manuscript. We would also like to thank Colin Lam for help with the flow cytometry data collection. Furthermore special thanks to the Joint Bioenergy Institute support staff for helping to make all experiment run faster and smoother. The funding for this work was provided by the Synthetic Biology Engineering Research Center (SynBERC), grant number 0540879, and by an NSF CAREER grant to M.M.M., grant number ECCS 0846618

\section{Author details}

${ }^{1}$ Department of Electrical Engineering and Computer Science, University of California, 656 Sutardja Dai Hall,Berkeley, Berkeley, CA94720, USA. ${ }^{2}$ California Institute for Quantitative Biosciences, University of California Berkeley, 5885 Hollis St, Emeryville, CA 94608, USA.

Received: 6 March 2012 Accepted: 14 June 2012

Published: 9 July 2012

\section{References}

1. Harris RE, Pargett M, Sutcliffe C, Umulis D, Ashe HL: Brat Promotes Stem Cell Differentiation via Control of a Bistable Switch that Restricts BMP Signaling. Developmental Cell 2011, 20:72-83.

2. F James $E \mathrm{Jr}$ : Self-perpetuating states in signal transduction: positive feedback, double-negative feedback and bistability. Current Opinion in Cell Biology 2002, 14:140-148.

3. Lai K, Robertson MJ, Schaffer DV: The Sonic Hedgehog Signaling System as a Bistable Genetic Switch. Biophysical Journal 2004, 86:2748-2757.

4. Wang L, Walker BL, lannaccone S, Bhatt D, Kennedy PJ, Tse WT: Bistable switches control memory and plasticity in cellular differentiation. Proceedings of the National Academy of Sciences 2009, 106:6638-6643.

5. Xiong W, Ferrell JE: A positive-feedback-based bistable//memory module/ ' that governs a cell fate decision. Nature 2003, 426:460-465.

6. Yan S-J, Zartman JJ, Zhang M, Scott A, Shvartsman SY, Li WX: Bistability coordinates activation of the EGFR and DPP pathways in Drosophila vein differentiation. Mol Syst Biol 2009, 5:278

7. Levskaya A, Chevalier AA, Tabor JJ, Simpson ZB, Lavery LA, Levy M, Davidson EA, Scouras A, Ellington AD, Marcotte EM, Voigt CA: Synthetic biology: Engineering Escherichia coli to see light. Nature 2005, 438:441-442.

8. Anderson JC, Clarke EJ, Arkin AP, Voigt CA: Environmentally Controlled Invasion of Cancer Cells by Engineered Bacteria. Journal of Molecular Biology 2006, 355:619-627.

9. Chen YY, Jensen MC, Smolke CD: Genetic control of mammalian T-cell proliferation with synthetic RNA regulatory systems. Proceedings of the National Academy of Sciences 2010, 107:8531-8536.

10. Chang D-E, Leung S, Atkinson MR, Reifler A, Forger D, Ninfa AJ: Building biological memory by linking positive feedback loops. Proceedings of the National Academy of Sciences 2010, 107:175-180.

11. Palani S, Sarkar CA: Synthetic conversion of a graded receptor signal into a tunable, reversible switch. Mol Syst Biol 2011, 7:480. 
12. Shah NA, Sarkar CA: Robust Network Topologies for Generating SwitchLike Cellular Responses. PLoS Comput Biol 2011, 7:e1002085.

13. Fritz G, Buchler NE, Hwa T, Gerland U: Designing sequential transcription logic: a simple genetic circuit for conditional memory. Systems and Synthetic Biology 2007, 1:89-98.

14. Gardner TS, Cantor CR, Collins JJ: Construction of a genetic toggle switch in Escherichia coli. Nature 2000, 403:339-342.

15. Tan C, Marguet P, You L: Emergent bistability by a growth-modulating positive feedback circuit. Nat Chem Biol 2009, 5:842-848.

16. Pfeuty B, Kaneko K: The combination of positive and negative feedback loops confers exquisite flexibility to biochemical switches. Physical Biology 2009, 6:046013.

17. Buchler NE, Gerland U, Hwa T: Nonlinear protein degradation and the function of genetic circuits. Proceedings of the National Academy of Sciences of the United States of America 2005, 102:9559-9564.

18. To T-L, Maheshri N: Noise Can Induce Bimodality in Positive Transcriptional Feedback Loops Without Bistability. Science 2010, 327:1142-1145.

19. Ochab-Marcinek A, Tabaka M: Bimodal gene expression in noncooperative regulatory systems. Proceedings of the National Academy of Sciences 2010, 107:22096-22101.

20. Pomerening JR, Sontag ED, Ferrell JE: Building a cell cycle oscillator: hysteresis and bistability in the activation of Cdc2. Nat Cell Biol 2003, 5:346-351

21. Santillán M, Mackey MC: Influence of Catabolite Repression and Inducer Exclusion on the Bistable Behavior of the lac Operon. Biophysical Journal 2004, 86:1282-1292.

22. Laurent M, Kellershohn N: Multistability: a major means of differentiation and evolution in biological systems. Trends in Biochemical Sciences 1999, 24:418-422.

23. Yildirim N, Mackey MC: Feedback Regulation in the Lactose Operon: A Mathematical Modeling Study and Comparison with Experimental Data. Biophysical Journal 2003, 84:2841-2851

24. Haseltine EL, Arnold FH: Implications of Rewiring Bacterial Quorum Sensing. Applied and Environmental Microbiology 2008, 74:437-445.

25. Gur E, Sauer RT: Evolution of the ssrA degradation tag in Mycoplasma: Specificity switch to a different protease. Proceedings of the National Academy of Sciences 2008, 105:16113-16118.

26. Gottesman S, Roche E, Zhou Y, Sauer RT: The ClpXP and ClpAP proteases degrade proteins with carboxy-terminal peptide tails added by the SsrA-tagging system. Genes \& Development 1998, 12:1338-1347.

27. Jenal U, Fuchs T: An essential protease involved in bacterial cell-cycle control. EMBO J 1998, 17:5658-5669.

28. Wiegert T, Schumann W: SsrA-Mediated Tagging in Bacillus subtilis. Journal of Bacteriology 2001, 183:3885-3889.

29. Griffith KL, Grossman AD: Inducible protein degradation in Bacillus subtilis using heterologous peptide tags and adaptor proteins to target substrates to the protease ClpXP. Molecular Microbiology 2008, 70:1012-1025.

30. Lutz R, Bujard H: Independent and Tight Regulation of Transcriptional Units in Escherichia Coli Via the LacR/O, the TetR/O and AraC/11-12 Regulatory Elements. Nucleic Acids Research 1997, 25:1203-1210.

31. Pedelacq J-D, Cabantous S, Tran T, Terwilliger TC, Waldo GS: Engineering and characterization of a superfolder green fluorescent protein. Nat Biotech 2006, 24:79-88.

32. Lou C, Liu X, Ni M, Huang Y, Huang Q, Huang L, Jiang L, Lu D, Wang M, Liu C, Chen D, Chen C, Chen X, Yang L, Ma H, Chen J, Ouyang Q: Synthesizing a novel genetic sequential logic circuit: a push-on push-off switch. Mol Syst Biol 2010, 6:350.

33. Hawley D, Johnson A, McClure W: Functional and physical characterization of transcription initiation complexes in the bacteriophage lambda OR region. J. Biol. Chem. 1985, 260:8618-8626

34. Part:BBa 112007 - partsregistry.org. http://partsregistry.org/wiki/index.php? title=Part:BBa_112007.

35. F James $E \mathrm{Jr}$ : Feedback regulation of opposing enzymes generates robust, all-or-none bistable responses. Current Biology 2008, 18:R244-R245.

36. Leeds JA, Beckwith J: Lambda repressor $\mathrm{N}$-terminal DNA-binding domain as an assay for protein transmembrane segment interactions in Vivo. Journal of Molecular Biology 1998, 280:799-810.

37. Tashiro Y, Fukutomi H, Terakubo K, Saito K, Umeno D: A nucleoside kinase as a dual selector for genetic switches and circuits. Nucleic Acids Research 2011, 39:e12.
38. Elowitz MB, Leibler S: A synthetic oscillatory network of transcriptional regulators. Nature 2000, 403:335-338.

39. Herman C, Thévenet D, Bouloc P, Walker GC, D'Ari R: Degradation of carboxy-terminal-tagged cytoplasmic proteins by the Escherichia coli protease HfIB (FtsH). Genes \& Development 1998, 12:1348-1355.

40. Basu S, Gerchman Y, Collins CH, Arnold FH, Weiss R: A synthetic multicellular system for programmed pattern formation. Nature 2005, 434:1130-1134.

41. Salis HM, Mirsky EA, Voigt CA: Automated design of synthetic ribosome binding sites to control protein expression. Nat Biotech 2009, 27:946-950.

42. Shaner NC, Campbell RE, Steinbach PA, Giepmans BNG, Palmer AE, Tsien RY: Improved monomeric red, orange and yellow fluorescent proteins derived from Discosoma sp. red fluorescent protein. Nat Biotech 2004, 22:1567-1572

43. Anderson JC, Dueber JE, Leguia M, Wu GC, Goler JA, Arkin AP, Keasling JD: BglBricks: A flexible standard for biological part assembly. J Biol Eng 2010, 4:1.

44. Round-the-horn site-directed mutagenesis - OpenWetWare. http:// openwetware.org/wiki/\%27Round-the-horn_site-directed_mutagenesis.

45. Keiler KC, Waller PR, Sauer RT: Role of a peptide tagging system in degradation of proteins synthesized from damaged messenger RNA. Science 1996, 271:990-993.

46. Potrykus K, Wegrzyn G, JHernandez V: Multiple Mechanisms of Transcription Inhibition by ppGpp at the $\lambda$ pR Promoter. Journal of Biological Chemistry 2002, 277:43785-43791.

47. Michalowski CB, Little JW: Positive Autoregulation of $\mathrm{Cl}$ Is a Dispensable Feature of the Phage $\lambda$ Gene Regulatory Circuitry. Journal of Bacteriology 2005, 187:6430-6442.

48. Meier I, Wray LV, Hillen W: Differential regulation of the Tn10---encoded tetracycline resistance genes tet $A$ and tetR by the tandem tet operators O1 and O2. The EMBO Journal 1988, 7:567-572.

49. Rauhut $R$, Klug G: mRNA Degradation in bacteria. FEMS Microbiology Reviews. 1999, 260:353-370.

50. Bintu L, Buchler NE, Garcia HG, Gerland U, Hwa T, Kondev J, Kuhlman T, Phillips R: Transcriptional regulation by the numbers: applications. Current Opinion in Genetics \& Development 2005,15:125-135.

51. Kubitschek HE, Friske JA: Determination of bacterial cell volume with the Coulter Counter. Journal of Bacteriology 1986,168:1466-1467.

doi:10.1186/1754-1611-6-9

Cite this article as: Huang et al: A genetic bistable switch utilizing nonlinear protein degradation. Journal of Biological Engineering 2012 6:9.

\section{Submit your next manuscript to BioMed Central and take full advantage of:}

- Convenient online submission

- Thorough peer review

- No space constraints or color figure charges

- Immediate publication on acceptance

- Inclusion in PubMed, CAS, Scopus and Google Scholar

- Research which is freely available for redistribution
Ciomed Central 